\title{
Cirurgia cardíaca no idoso
}

\author{
Danton R. da Rocha LOURES* ${ }^{*}$, Roberto Gomes de CARVALHO*, Leonardo MULINARI*, \\ Arleto Zacarias SILVA Jr. ${ }^{*}$, Carlos Augusto SCHMIDLIN*, Maricélia BROMMELSTRÖET**, \\ Vinícius Nicolau VOITOWICZ*, Marcelo Haddad DANTAS*, Ricardo José CHOMA*, Sérgio SHIBATA*, \\ Marcello Laneza FELICIO*, Dênis BONATTO*, Nilo ANTUNES FILHO*
}

RBCCV 44205-480

Loures D R R, Carvalho R G, Mulinari L, Silva Jr. A Z, Schmidlin C A, Brommelströet M, Voitowicz V N, Dantas M $\mathrm{H}$, Choma R J, Shibata S, Felício M L, Bonatto D, Antunes Filho N - Cirurgia cardíaca no idoso. Rev Bras Cir Cardiovasc 2000; 15 (1): 1-5.

RESUMO: Com o aumento da expectativa de vida da população brasileira cresce o número de pessoas com idade superior a 70 anos que necessitam de operação cardíaca.

Casuística e Métodos: Foram avaliados, retrospectivamente, 75 pacientes com idade ${ }^{3}$ a 70 anos submetidos a operação cardíaca no HC-UFPR, entre 1995 e 1999, com objetivo de analisarmos os resultados imediatos e tardios. A idade variou de 70 a 88 anos, sendo $34(46,7 \%)$ do sexo feminino e $41(53,3 \%)$ do masculino. Os principais sintomas foram angina $(81,3 \%)$, dispnéia $(42,6 \%)$ e síncope $(16 \%)$. Os pacientes encontravam-se em classe I (57,3\%), classe II (17,3\%), classe III (18,6\%) e classe IV $(6,6 \%)$ da NYHA, $61,3 \%$ eram hipertensos, $48 \%$ tabagistas, $28 \%$ diabéticos e $9,3 \%$ haviam sido submetidos a operação cardíaca prévia. Foram realizadas $50(66,6 \%)$ revascularizações do miocárdio, $9(12 \%)$ trocas de valva aórtica, $5(6,6 \%)$ operações de aorta, 4 (5,2\%) trocas valvares + revascularização miocárdica e outros procedimentos (7\%). As principais complicações pós-operatórias foram cardiovasculares: arritmias ventriculares $(22,6 \%)$, arritmias supraventriculares $(21,3 \%)$, baixo débito cardíaco (16\%); infecciosas $(16 \%)$ e pulmonares $(9,3 \%)$. O tempo médio de permanência na UTI foi de 5 dias.

Resultados: A mortalidade hospitalar foi de $13,3 \%$ e houve 5 óbitos tardios. Dos sobreviventes, $78,4 \%$ compareceram para seguimento ambulatorial. O tempo médio de seguimento foi de 20,7 meses e a sobrevida foi de $92 \%$; um dos óbitos tardios foi de origem cardiovascular.

Conclusão: Apesar de serem pacientes de maior complexidade clínica pela maior incidência de doenças crônicas e acometimento de outros órgãos, os avanços na cirurgia cardíaca e terapia intensiva tornaram possível a intervenção com baixa morbi-mortalidade.

DESCRITORES: Envelhecimento, fisiologia. Procedimentos cirúrgicos cardíacos. Cardiopatias, cirurgia, idoso. Cirurgia cardíaca, idoso.

\section{INTRODUÇÃO}

Com o crescente aumento da expectativa de vida da população brasileira, é cada vez maior o número de pessoas a atingir os 70 anos de idade e que necessite de algum tipo de intervenção cardiovascular. Segundo fontes do IBGE, no Brasil a população de idosos já atinge cerca de $7 \mathrm{mi}$ Ihões, devendo ter um aumento progressivo na atual velocidade de crescimento ( $3,5 \%$ ao ano). A expectativa de vida do brasileiro ao nascer em 1940 era de 41,5 anos. Os maiores ganhos de expectativa de vida ocorreram na década de 80 , quando aumentou de 53,5 anos de idade em 1970 para 61,8 anos de idade em 1980, sendo atualmente de 67,6 anos. Esta é uma tendência observada nas populações ocidentais. Estudos realizados nos Estados Unidos, no início da década de 80 , mostraram que

Trabalho realizado no Hospital de Clínicas da Universidade Federal do Paraná. Curitiba, PR, Brasil.

Recebido para publicação em setembro de 1999.

* Do Serviço de Cirurgia Torácica e Cardiovascular do Hospital de Clínicas da UFPR.

Endereço para correspondência: Danton R. da Rocha Loures. Rua General Carneiro, 181. $7^{\circ}$ andar, sala 704. Bairro Alto da Glória. Curitiba, PR, Brasil. CEP: 80060-900. Tel: (041) 360-1800. Fax: (041) 264-5872. 
Loures D R R, Carvalho R G, Mulinari L, Silva Jr. A Z, Schmidlin C A, Brommelströet M, Voitowicz V N, Dantas M H, Choma R J, Shibata

S, Felício M L, Bonatto D, Antunes Filho N - Cirurgia cardíaca no idoso. $\quad$ Rev Bras Cir Cardiovasc 2000; 15 (1):1-5.

$40 \%$ dos norte-americanos atingirão 80 anos de idade e que os idosos irão compor cerca de 20 a $25 \%$ de sua população, na primeira metade do próximo século (1-4). A doença cardiovascular é extremamente prevalente nesta faixa etária, com aproximadamente $40 \%$ dos octogenários apresentando doença cardiovascular sintomática. A incidência de doença coronariana obstrutiva em pessoas com idade superior a 70 anos chega a $76 \%$, sendo comum a associação com doença valvar (4-6).

Este estudo tem o objetivo de avaliar os resultados imediatos e tardios das operações cardíacas realizadas em pacientes com idade igual ou superior a 70 anos, no período entre 1995 e 1999, no Serviço de Cirurgia Torácica e Cardiovascular do Hospital de Clínicas da Universidade Federal do Paraná (HC-UFPR).

\section{CASUÍSTICA E MÉTODOS}

Foram avaliados, retrospectivamente, 75 pacientes com idade 3 a 70 anos submetidos a operação cardiovascular no Serviço de Cirurgia Torácica e Cardiovascular do HC-UFPR, entre janeiro de 1995 e janeiro de 1999. A idade dos pacientes variou de 70 a 88 anos (média: 74,5 anos), sendo 41 (53,3\%) do sexo masculino e $34(46,7 \%)$ do feminino. As principais características clínicas do grupo de pacientes estudados são apresentadas na Tabela 1.

Foram considerados hipertensos os pacientes que estavam recebendo algum tipo de medicação antihipertensiva e portadores de dislipidemias os que apresentavam dosagem de colesterol total $3200 \mathrm{mg} / \mathrm{dl}$. Doença vascular periférica foi considerada presente em pacientes com história de claudicação intermitente, operação vascular periférica ou ambos. Pacientes com doença cerebrovascular incluíram aqueles com história de acidente vascular cerebral (AVC), ataque isquêmico transitório (AIT) e sopro carotídeo confirmado por ecodoppler. Insuficiência renal pré-operatória foi considerada em pacientes com níveis de creatinina sérica 3 1,5 mg/dl. Considerou-se como infarto agudo do miocárdio (IAM) prévio o ocorrido até, pelo menos, quinze dias antes da operação. Todos os pacientes foram submetidos a cineangiocoronariografia pré-operatória.

Os procedimentos cirúrgicos realizados estão apresentados na Tabela 2.

Após anestesia geral, era realizada esternotomia mediana, eventualmente toracotomia póstero-lateral nas operações de aorta descendente, canulação da aorta e átrio direito ou bicaval. Em seguida, era realizada a heparinização sistêmica e iniciada CEC com oxigenadores de membrana, perfusão nãopulsátil e hipotermia moderada. A proteção miocár-

\section{TABELA 1}

CARACTERÍSTICAS CLÍNICAS DOS PACIENTES ESTUDADOS

\begin{tabular}{|c|c|}
\hline VARIÁVEL & $N^{\circ}$ DE PACIENTES \\
\hline \multicolumn{2}{|l|}{ Idade } \\
\hline Média & 74,5 \\
\hline Variação & $70-88$ \\
\hline Sexo (masculino/feminino) & $41 / 34$ \\
\hline Angina & $61 \quad(81,3 \%)$ \\
\hline \multicolumn{2}{|l|}{ ICC - NYHA* } \\
\hline classe I & $43(57,3 \%)$ \\
\hline classe II & $13(17,3 \%)$ \\
\hline classe III & $14(18,6 \%)$ \\
\hline classe IV & $5 \quad(6,6 \%)$ \\
\hline Síncope & $12 \quad(16 \%)$ \\
\hline $\mathrm{HAS}^{* *}$ & $46 \quad(61,3 \%)$ \\
\hline Tabagismo & $36 \quad(48 \%)$ \\
\hline $\mathrm{IAM}^{* * *}$ & $(32 \%)$ \\
\hline Diabete melito & $21 \quad(28 \%)$ \\
\hline Dislipidemias & $14(18,6 \%)$ \\
\hline Doença vascular periférica & $8(10,6 \%)$ \\
\hline Doença cerebrovascular & $8(10,6 \%)$ \\
\hline Neoplasia & $5 \quad(6,6 \%)$ \\
\hline Insuficiência renal & $4 \quad(5,3 \%)$ \\
\hline Operação cardíaca prévia & $7 \quad(9,3 \%)$ \\
\hline
\end{tabular}

* ICC-NYHA - classificação funcional para insuficiência cardíaca segundo a New York Heart Association; ${ }^{* *}$ HAS - hipertensão arterial sistêmica; ${ }^{* * *}$ IAM - infarto agudo do miocárdio.

dica era obtida com solução cardioplégica sangüínea hipotérmica anterógrada. Nos pacientes valvares houve preferência pela utilização de próteses biológicas e nos coronarianos utilização de enxertos de artéria torácica interna esquerda para o ramo descendente anterior e de veia safena para os demais ramos coronarianos com lesões críticas. O número de enxertos realizados era definido pelo número de anastomoses distais.

As intercorrências pós-operatórias foram separadas em complicações cardiovasculares - arritmias cardíacas, baixo débito cardíaco, ICC, IAM, choque cardiogênico, choque hipovolêmico - infecciosas, pulmonares, neurológicas e renais. As arritmias cardíacas e os transtornos de condução eram detectadas pelo cardioscópio e eletrocardiograma (ECG) e foram consideradas quando levaram à instabilidade hemodinâmica e necessidade de reversão farmacológica ou elétrica, ou de estimulação cardíaca artificial temporária. A presença de baixo débito cardíaco foi considerada nos pacientes com instabilidade hemodinâmica havendo necessidade de drogas vasoativas por tempo superior a 48h. A ocorrência de infecção era verificada pelo quadro clínico, exames laboratoriais e necessidade de antibioticoterapia prolongada, AVC pelas alterações neurológicas localizadas ou de nível de consciência por mais de $24 \mathrm{~h}$. Os pacientes com 
Loures D R R, Carvalho R G, Mulinari L, Silva Jr. A Z, Schmidlin C A, Brommelströet M, Voitowicz V N, Dantas M H, Choma R J, Shibata

S, Felício M L, Bonatto D, Antunes Filho N - Cirurgia cardíaca no idoso. $\quad$ Rev Bras Cir Cardiovasc 2000; 15 (1):1-5.

TABELA 2

PROCEDIMENTOS CIRÚRGICOS REALIZADOS E VARIÁVEIS TRANSOPERATÓRIAS

VARIÁVEL

$N^{\circ}$ DE PACIENTES

\begin{tabular}{|c|c|}
\hline Revascularização do miocárdio & $50(66,6 \%)$ \\
\hline Troca de valva aórtica & $9(12 \%)$ \\
\hline Operação de aorta & $5(6,6 \%)$ \\
\hline Revascularização do miocárdio + aneurismectomia de VE* & $3(4 \%)$ \\
\hline Revascularização do miocárdio + troca de valva aórtica & $2(2,6 \%)$ \\
\hline Revascularização do miocárdio + troca de valva mitral & $2(2,6 \%)$ \\
\hline Troca de valva aórtica + aorta ascendente & $1(1,3 \%)$ \\
\hline Plastia de valva mitral & $1(1,3 \%)$ \\
\hline Plastia de valva tricúspide & $1(1,3 \%)$ \\
\hline Fechamento de $\mathrm{CIA}^{\star *}$ & $1(1,3 \%)$ \\
\hline \multicolumn{2}{|l|}{ Tempo de $\mathrm{CEC}^{* * *}(\mathrm{~min})$} \\
\hline média & 100 \\
\hline variação & 48-176 \\
\hline \multicolumn{2}{|l|}{ Tempo de pinçamento aórtico (min) } \\
\hline média & 53 \\
\hline variação & $5-109$ \\
\hline \multicolumn{2}{|l|}{ Tipo de prótese valvar } \\
\hline biológica & $14(100 \%)$ \\
\hline \multicolumn{2}{|l|}{ Número de enxertos/paciente } \\
\hline média & 2,4 \\
\hline variação & $1-4$ \\
\hline
\end{tabular}

VE: ventrículo esquerdo; ${ }^{* *}$ CIA: comunicação interatrial; *** CEC: circulação extracorpórea.

nível sérico de creatinina $32,0 \mathrm{mg} / \mathrm{dl}$ no pós-operatório foram considerados como portadores de insuficiência renal. As complicações pulmonares incluíram todas aquelas que levaram a ventilação mecânica prolongada. Todos os óbitos ocorridos durante a internação ou nos primeiros 30 dias após a operação foram considerados óbitos hospitalares.

O "follow up" dos pacientes foi obtido através de consulta ambulatorial e contato telefônico, quando necessário.

\section{RESULTADOS}

$\mathrm{Na}$ Tabela 3 são apresentadas as principais intercorrências pós-operatórias.

A mortalidade hospitalar foi de $13,3 \%$, a causa dos óbitos foi choque cardiogênico em 5 pacientes, IAM em 2, choque hipovolêmico em 2 e infecciosa (mediastinite) em 1 paciente.

Dos 65 pacientes que sobreviveram, compareceram $51(78,4 \%)$ para seguimento ambulatorial. O tempo de seguimento variou de 1 a 48 meses (média: 20,7 meses). Houve 5 óbitos tardios, sendo 2 de origem neoplásica, 1 por hemorragia digestiva alta, 1 de etiologia infecciosa (mediastinite) e 1 de origem cardiovascular (IAM). A sobrevida tardia foi de $92 \%$.

\section{COMENTÁRIOS}

O aumento da expectativa de vida da população brasileira trouxe consigo as enfermidades cardiovasculares inerentes à oitava década de vida, como a doença valvar aórtica devido às alterações degenerativas de fibrose e calcificação valvar $(7,8)$ e a

TABELA 3

\begin{tabular}{lc}
\hline INCIDÊNCIA DE COMPLICAÇÕES PÓS-OPERATÓRIAS \\
\hline COMPLICAÇÃO & $N^{\circ}$ DE PACIENTES \\
\hline Cardiovascular & \\
Arritmia ventricular & $17(22,6 \%)$ \\
Arritmia supraventricular & $16(21,3 \%)$ \\
Baixo débito cardíaco & $12(16 \%)$ \\
ICC & $6(8 \%)$ \\
IAM & $5(6,6 \%)$ \\
Choque cardiogênico & $5(6,6 \%)$ \\
Choque hipovolêmico & $3(4 \%)$ \\
Reoperação por sangramento & $3(4 \%)$ \\
Transtorno de condução & $1(1,3 \%)$ \\
Infecciosas & $12(16 \%)$ \\
Pulmonares & $7(9,3 \%)$ \\
AVC & $4(5,3 \%)$ \\
\hline Insuficiência renal & $4(5,3 \%)$ \\
Óbitos & $10(13,3 \%)$ \\
\hline
\end{tabular}


Loures D R R, Carvalho R G, Mulinari L, Silva Jr. A Z, Schmidlin C A, Brommelströet M, Voitowicz V N, Dantas M H, Choma R J, Shibata

S, Felício M L, Bonatto D, Antunes Filho N - Cirurgia cardíaca no idoso. $\quad$ Rev Bras Cir Cardiovasc 2000; 15 (1):1-5.

doença coronariana obstrutiva, cuja incidência aumenta dramaticamente com a idade. Dos 40 aos 49 anos a incidência de doença coronariana obstrutiva severa é de $33 \%$, dos 50 aos 59 é de $38 \%$, dos 60 aos 69 é de $64 \%$ e acima dos 70 anos chega a $76 \%$, sendo comum a associação com valvopatias (5). Diversos modelos de estudo epidemiológico prevêem que, com as atuais taxas de crescimento da população idosa, haverá não só um aumento na incidência de doença arterial coronariana, mas também uma elevação progressiva do número de pacientes idosos que se apresentam aos Serviços de cirurgia cardiovascular (1).

A decisão de submeter o paciente idoso à intervenção cirúrgica depende da relação risco-benefício baseada em fatores dentre os quais estão a classe funcional, a estabilidade emocional e a qualidade geral de vida. Além do mais, freqüentemente há discrepância entre a idade e o estado geral do paciente, devendo-se utilizar como critério para a escolha do tratamento o estado fisiológico do indivíduo, em vez de sua idade cronológica, evitando retardar o momento da operação, pois sua realização deve ser anterior ao aparecimento de disfunção miocárdica severa (4-6).

Apesar de serem pacientes de maior complexidade clínica devido à maior incidência de doenças crônicas e de acometimento de outros órgãos, os avanços na cirurgia cardíaca, anestesia, circulação

extracorpórea e terapia intensiva têm contribuído para a intervenção com baixa morbi-mortalidade nesta população, demonstrando segurança e eficácia da operação cardíaca não apenas nos septuagenários bem como nos octogenários $(3,5,6,8)$. No presente estudo, a mortalidade hospitalar foi de $13,3 \%$ e encontra-se dentro dos índices relatados na literatura (1-8). Vários autores têm recomendado que um meticuloso e agressivo controle pós-operatório deve ser oferecido a estes pacientes no sentido de minimizar as complicações pós-operatórias. Atenção especial deve ser dada à mobilização precoce, tratamento das arritmias, evitar uso de drogas nefrotóxicas, manutenção de um adequado estado nutricional, prevenção de complicações infecciosas pela retirada de cateteres e drenos. Todos esses cuidados podem levar a resultados próximos àqueles obtidos nas populações mais jovens $(3,6,8,9)$.

\section{CONCLUSÃO}

Apesar dos pacientes com idade ${ }^{3}$ a 70 anos
apresentarem maior complexidade clínica devido a
maior incidência de doenças crônicas e de com-
prometimento de órgãos-alvo, os avanços na ope-
ração cardíaca, anestesia e terapia intensiva, bem
como a indicação cirúrgica antes de que haja
disfunção miocárdica severa, tornaram possível a
intervenção cirúrgica com baixa morbi-mortalidade.

RBCCV 44205-480

Loures D R R, Carvalho R G, Mulinari L, Silva Jr. A Z, Schmidlin C A, Brommelströet M, Voitowicz V N, Dantas $\mathrm{M} \mathrm{H}$, Choma R J, Shibata S, Felício M L, Bonatto D, Antunes Filho N - Cardiac surgery in elderly patients. Rev Bras Cir Cardiovasc 2000; 15 (1): 1-5

ABSTRACT: Background: Because of the increase in the life expectancy of the Brazilian population, elderly patients are being increasingly referred to cardiac surgery.

Material and Methods: Seventy-five patients, 70 years of age or older, who underwent cardiac surgery in the HC-UFPR, between 1995 and 1999 were studied. The purpose of this study was to analyze early and longterm results. Ages from 70 to 88 years, with 34 females (46.7\%) and 41 males (53.3\%). Symptoms included angina $(81.3 \%)$, dyspnea $(42.6 \%)$ and syncope $(16 \%)$. There was $57.3 \%$ of patients in NYHA class I, $17.3 \%$ in class II, $18.6 \%$ in class III and $6.6 \%$ in class IV. The main risk factors listed were high blood pressure $(61.3 \%)$ smoke $(48 \%)$, diabetes mellitus $(28 \%)$ and $9.3 \%$ had already had cardiac operations. Surgical procedures included 50 coronary artery bypass grafts - CABG (66.6\%), 9 aortic valve replacements (12\%), 5 aortic operations $(6.6 \%), 4 \mathrm{CABG}+$ valve replacement $(5.2 \%)$ and other procedures $(7 \%)$. The main postoperative complications were cardiovascular - ventricular arrhythmias (22.6\%), supraventricular arrhythmias (21.3\%), low cardiac output (16\%) - infections (16\%) and pulmonary problems $(9.3 \%)$. The median stay in the ICU was 5 days

Results: Sixty-five (78.4\%) survivors had complete follow-up. The mean follow-up time was 20.7 months and long-term survival was $92 \%$. Only one of the late deaths was cardiac related.

Conclusion: Although this subgroup is associated with chronic diseases compromising other organs, advances in cardiac surgery and intensive care have made possible an intervention with safety and low morbidity and mortality.

DESCRIPTORS: Aging, physiology. Cardiac surgical procedures. Heart disease, surgery. Cardiac surgical procedures, aged. 


\section{REFERÊNCIAS BIBLIOGRÁFICAS}

1 Iglezias J C R, Oliveira Jr. J L, Fels K W et al. - Fatores prognósticos na revascularização do miocárdio em pacientes idosos. Rev Bras Cir Cardiovasc 1997; 12: 325-34.

2 Chocron S, Etievent J Ph, Clement F et al. - Is surgery for aortic stenosis justified in patients over 75 years of age? J Cardiovasc Surg 1996; 37: 255-9.

3 Adkins M S, Amalfitano D, Harnum N A, Laub G W, McGrath L B - Efficacy of combined coronary revascularization and valve procedures in octogenarians. Chest 1995; 108: 927-31.

4 Gehlot A, Mullany C J, Ilstrup D et al. - Aortic valve replacement in patients aged eighty years and older: early and long-term results. $J$ Thorac Cardiovasc Surg 1996; 111: 1026-36.

5 Kirsch M, Guesnier L, LeBesnerais $\mathrm{P}$ et al. - Cardiac operations in octogenarians: perioperative risk factors for death and impaired autonomy. Ann Thorac Surg 1998; 66: 60-7.

6 Elayda M A, Hall R J, Reul R M et al. - Aortic valve replacement in patients 80 years and older: operative risks and long-term results. Circulation 1993; 88 (5 Pt 2): 11-6.

7 Abdalla L A, Piegas L S, Timerman S et al. - Manuseio cirúrgico da valva aórtica em pacientes acima de 70 anos. Arq Bras Cardiol 1992; 58: 453-5.

8 Aranki S F, Rizzo R J, Couper G S et al. - Aortic valve replacement in the elderly: effect of gender and coronary artery disease on operative mortality. Circulation 1993; 88 (5 Pt 2): 17-23.

9 Roques X F \& Baudet E M - Long term results of cardiac valve replacement in patients aged 75 years and older. J Cardiovasc Surg 1991; 32: 794-9. 